\title{
EDITORIAL
}

\section{Telemedicina: um pouco de história antes do crescimento exponencial durante a pandemia de Covid-19}

\author{
Telemedicine: brief history before exponential growth during Covid-19 pandemic
}

\author{
Carlos H. S. Pedrotti, Tarso A. D. Accorsi, Karine De Amicis Lima, \\ Renata A. Morbeck, Eduardo Cordioli
}

\begin{abstract}
"O ano que não terminou". Assim é denominado o ano de 1968, em que diversos acontecimentos políticos no mundo como o assassinato de Martin Luther King Jr. e do presidente Robert Kennedy, Guerra do Vietnã, instituição do AI-5 no Brasil e muitos outros eventos políticos acabavam por obscurecer o enorme desenvolvimento tecnológico decorrente da corrida espacial e avanços nas telecomunicações por satélites. Na década de 60 surgiram os primeiros registros do uso de informática para fins comerciais, o lançamento do chip pela IBM e a ARPANET (Advanced Research Projects Agency Network), o embrião da internet atual' ${ }^{1}$.

Embalados pelo famoso desenho animado "Os Jetsons", passado em 2062 e que mostrava carros voadores, robôs faxineiros e muitas videochamadas, os pesquisadores Raymond Murphy Jr. e Kenneth Bird, da Universidade de Harvard, foram os primeiros a demonstrar em escala o potencial da telemedicina por vídeo como forma alternativa de atender pacientes ${ }^{2}$. De agosto de 1968 a dezembro de 1969, mil pacientes (sim, 1000 pacientes!) atendidos no ambulatório do movimentado aeroporto internacional de Boston, nos Estados Unidos, passaram por consultas remotas utilizando um precário circuito interno de TV em contato com o Hospital Geral de Massachusetts, a 4,5 $\mathrm{km}$ de distância, intermediados por um profissional de enfermagem no local.

O artigo relatando o experimento, publicado em 1974, é emblemático. Descreve com detalhes caraterísticas do exame físico realizado pelo vídeo, o elevado grau de acurácia diagnóstica em situações de baixa complexidade e alta satisfação do paciente. Concluem que o telediagnóstico é factível e, em tradução literal, relatam: "A tecnologia está disponivel atualmente. Sistema deste tipo podem levar conhecimentos e técnicas especializadas a áreas remotas ou nem tão remotas, mas medicamente desfavorecidas"2. É conveniente lembrar que a resolução da imagem era de 480 × 320, menos de $25 \%$ da pior resolução HD atual, e era em preto e branco (!).

Mais de 50 anos se passaram e a implementação de projetos semelhantes, de abrangência e escala maiores, se mostrou mais complexo do que inicialmente imaginado. O custo de implantação de circuitos de TV à época não justificava os investimentos, além de permitir apenas comunicação em duas vias, sem possibilidade de transferências de chamadas. Na década de 70 e 80 vemos apenas experimentos de escala local, com evolução tecnológica prejudicada por sucessivas crises financeiras.
\end{abstract}

Centro de Telemedicina, Hospital Israelita Albert Einstein, São Paulo, SP, Brasil. ORCID: Pedrotti CHS - https://orcid.org/0000-00020634-7086; Accorsi TAD - https://orcid.org/0000-0002-8023-3466; Lima KA - https://orcid.org/0000-0002-9936-2436; Morbeck RA - https://orcid.org/0000-0003-1789-1738; Cordioli E - https://orcid.org/0000-0001-5405-9380. Email: carlos.pedrotti@einstein.br, tarsoa@einstein.br, karineamicis@gmail.com, renata.albaladejo@einstein.br, eduardo.cordioli@einstein.br.

Endereço para correspondência: Carlos H. S Pedrotti. Secretaria do Centro de Telemedicina. Av. Albert Einstein, 627. Bloco B, $2^{\circ}$ andar. São Paulo, SP. CEP: 05652-900. Email: carlos.pedrotti@einstein.br. 
Na década de 90 a Georgia, estado do sudeste dos Estados Unidos com economia predominantemente rural, financiou um grande projeto piloto de telemedicina para ampliar o acesso à saúde. Onze de seus 159 municípios não tinha nenhum médico e mais de $50 \%$ não tinha pediatras ${ }^{3}$. Em parceria com empresas de telecomunicação, o projeto ganhou escala em meados de 2006 e em 2010 já tinha atendido mais de 30 mil pacientes e gerou conhecimento inestimável para o avanço da telemedicina no mundo todo ${ }^{4}$.

Apesar da telemedicina entre médicos e pacientes, intermediados ou não por outros profissionais de saúde, realizada por radiofrequência ou telefonia já ocorrer desde o início do século XX, a adição do vídeo rompe um novo paradigma na acurácia diagnóstica e relação entre médico e paciente. De fato, estudos clássicos realizados desde a década de 60 mostram que a visualização das expressões faciais e obtenção de elementos visuais não verbais são reveladores e podem ser responsáveis por até $55 \%$ dos componentes da comunicação entre seres humanos ${ }^{5}$. Na medicina, a comunicação não verbal é elemento importante da relação médico-paciente ${ }^{6} \mathrm{e}$ a anamnese pode contribuir por mais de $80 \%$ da investigação diagnóstica e terapêutica ${ }^{7}$, sendo que um bom exame físico visual possivelmente ainda acrescentaria parte significativa da contribuição do exame clínico, ainda não quantificada objetivamente.

O desenvolvimento das mídias digitais, a internet e mais recentemente a ampla disponibilidade de banda larga propiciaram avanços nas formas de comunicação audiovisual. A taxa de crescimento de 2005 a 2017 do número de visitas foi de $52 \%$ ano a ano ${ }^{8}$, estimando-se que em 2019 possa ter atingido a marca de 50 milhões de teleconsultas apenas nos Estados Unidos.

Embora utilizada nas mais diversas especialidades, os maiores crescimentos são observados na atenção primária em doenças crônicas ou de rotina, e na avaliação de situações clínicas agudas de baixa complexidade. E é especificamente esta última a responsável pelo ponto de inflexão observado em março de 2020, junto à pandemia de Covid-19, iniciando um ciclo exponencial de adoção da tecnologia em todo o mundo. Previsões estimam que o número de interações médicas por telemedicina em 2020 possa chegar a mais de 1 bilhão, incluindo 900 milhões de consultas relacionadas a Covid-199.

Surgem assim inúmeros questionamentos quanto à qualidade do cuidado prestado à distância, pela internet, incluindo potenciais prejuízos ao diagnóstico, relação médico-paciente e manejo inadequado dos casos, podendo levar a prejuízos ao prognóstico. Ocorre que inúmeros estudos e metanálises tem mostrado que a consulta por telemedicina traz resultados comparáveis ao atendimento presencial, alta satisfação do paciente e pode resolver até $80 \%$ dos casos sem a necessidade de avaliação face a face. Tal comparabilidade vem acompanhada de inúmeros benefícios, como custos inferiores, redução e deslocamentos, maior acesso a uma população desfavorecida logisticamente e, obviamente, um acesso seguro quando indicado isolamento social ${ }^{10}$.

O raciocínio diagnóstico, componente fundamental do pensamento médico, inicia-se habitualmente com o "ouvir ao paciente", o que implica a formação das probabilidades iniciais. A extração de outras informações na anamnese, seja através do reconhecimento de padrões ou técnica dedutiva, fornece elementos que vão influenciar positiva ou negativamente as probabilidades iniciais, isto é, originar probabilidades pós-teste. Sequencialmente, novas informações vão modificando as probabilidades, até uma ou mais possibilidades diagnósticas maiores restarem, ou melhor, um ou mais diagnósticos serem presumidos.

O exame físico e exames complementares não são mera parte burocrática da ação médica, mas sim etapas que provêm novas informações para a sequência do pensamento exposto. Inúmeras hipóteses diagnósticas têm sua probabilidade nada ou pouco influenciada por avaliação física e outros exames complementares. Mais importante que o dogma da avaliação presencial é o pensamento médico. Se o julgamento clínico de uma avaliação remota implicar alta probabilidade diagnóstica sem necessidade de mais exames ou testes, trata-se de situação em que a telemedicina tem o mesmo benefício presumido que avaliação presencial. Destaca-se que a qualidade do fluxo diagnóstico por avaliação remota tem vantagens outras como, por exemplo, o ambiente mais confortável e menos estressante. Tanto para paciente quanto para equipe médica, a redução do desconforto e estresse do deslocamento e salas de espera provavelmente aumentam a acurácia diagnóstica da anamnese, assim como permitem que o profissional faça laços maiores de confiança com o paciente, demonstre empatia e tenha mais tempo para explicações, sem interrupções de diversas naturezas.

A teleconsulta não tem a pretensão de substituir a avaliação tradicional e, obviamente, diversas situações principalmente àquelas com ameaça à vida - cujas hipóteses e/ou tratamentos não são seguramente estabelecidos apenas com as informações obtidas, deverão ser referenciadas para avaliação presencial e com mais recursos. Não se pode deixar de ressaltar um potencial interessante da telemedicina: disseminar informações de qualidade de 
forma fácil e com baixo-custo, informações emitidas por profissionais de saúde que voltam a ter protagonismo social, maior acolhimento e inserção dos pacientes no sistema de saúde, aumentando a parceria médico-paciente e provavelmente cursando com uso racional de recursos.

No entanto, ainda há muitos desafios a serem superados. Os limites exatos da tecnologia ainda estão sendo definidos e uma gigantesca quantidade de dados será gerada, analisada e publicada por serviços de telemedicina que estão crescendo em todo o mundo. Uma rápida busca na internet mostra dezenas de artigos já publicados nas últimas semanas, revisando os potenciais dos atendimentos à distância, em múltiplas especialidades, durante a epidemia. E, fora deste momento epidemiológico, já é extremamente vasta a quantidade de artigos com evidência de qualidade publicados, mostrando a segurança e eficácia da telemedicina nos mais variados cenários.

Já é consenso que a adoção da telemedicina atingiu um novo patamar e que, mesmo com o fim da pandemia, a utilização de meios eletrônicos para a relação entre médicos e pacientes, além de todos os outros profissionais envolvidos nos cuidados à saúde, crescerá de forma sustentada em todas as áreas da medicina. A disseminação que a telemedicina é eficaz e a melhor compreensão de seus limites, assim como maior integração do mundo digital às estruturas físicas de saúde, trarão maior acesso à saúde para todos e garantirá maior sustentabilidade dos sistemas de saúde ao redor do globo.

O ano de 1968 pode ter mudado o mundo para sempre, mas foi superado há muito tempo. A ficção científica das videochamadas mostradas nos "Jetsons" já é realidade acessível a grande parte da população, e está sendo aplicada com sucesso na atenção à saúde. O objetivo primordial da boa medicina é, e sempre será, o bem-estar do paciente, a segurança e a qualidade do cuidado, embasados na ciência, praticados com humanismo e empatia. Isso nenhuma nova tecnologia mudará. A telemedicina não altera este objetivo, mas sim ajuda a atingi-lo.

Conflito de Interesses: Os autores declaram não possuir conflito de interesses relacionados ao presente manuscrito.

Participação dos autores: Pedrotti CHS, Accorsi TAD: redação, revisão. Lima KA, Morbeck RA, Cordioli E: revisão e aprovação.

\section{REFERÊNCIAS}

1. Salus PH, Cerf VG. Casting the Net: from ARPANET to internet and beyond. Boston: Addison-Wesley Longman Publishing Co., Inc., USA; 1995.

2. Murphy RL Jr, Bird KT. Telediagnosis: a new community health resource. Observations on the feasibility of telediagnosis based on 1000 patient transactions. Am J Public Health. 1974;64(2):113-9. doi: http://dx.doi.org/10.2105/ajph.64.2.113.

3. Adams LN, Grigsby RK. The Georgia State Telemedicine Program: initiation, design, and plans. Telemed J. 1995;1(3):22735. doi: http://dx.doi.org/10.1089/tmj.1.1995.1.227.

4. Brewer R, Goble G, Guy P. A peach of a telehealth program: Georgia connects rural communities to better healthcare. Perspect Health Inf Manag. 2011;8(Winter):1c. Available from: https://www.ncbi.nlm.nih.gov/pmc/articles/PMC3035827/.

5. Mehrabian A. Silent messages. Belmont: Wadsworth Publishing Company, Inc, USA; 1971.

6. Mast MS. On the importance of nonverbal communication in the physician-patient interaction. Patient Educ Couns. 2007;67(3):315-8. doi: http://dx.doi.org/10.1016/j.pec.2007.03.005.

7. Hampton JR, Harrison MJ, Mitchell JR, Prichard JS, Seymour C. Relative contributions of history-taking, physical examination, and laboratory investigation to diagnosis and management of medical outpatients. Br Med J. 1975;2(5969):4869. doi: http://dx.doi.org/10.1136/bmj.2.5969.486.

8. Barnett ML, Ray KN, Souza J, Mehrotra A. Trends in telemedicine use in a large commercially isured population, 20052017. JAMA. 2018;320(20):2147-9. doi: http://dx.doi.org/10.1001/jama.2018.12354.

9. Forrester Research, Inc. US virtual care visits to soar to more than 1 billion [cited 2020 Aug 14]. Available from: https:// go.forrester.com/press-newsroom/us-virtual-care-visits-to-soar-to-more-than-1-billion/.

10. Hollander JE, Carr BG. Virtually perfect? Telemedicine for Covid-19. N Engl J Med. 2020;382(18):1679-81. doi: http:// dx.doi.org/10.1056/NEJMp2003539. 